\title{
State of the art in breast intraoperative electron radiation therapy after intraoperative ultrasound introduction
}

\author{
Cristiana Vidali ${ }^{1}$, Mara Severgnini² ${ }^{2}$ Gabriele Bellio ${ }^{3}$, Fabiola Giudici ${ }^{4}$, Vittorino Milan ${ }^{5}$, \\ Zaira Pellin ${ }^{3}$, Sara Savatovic ${ }^{6}$, Serena Scomersi ${ }^{3}$, Gerd Fastner ${ }^{7}$, Antonella Ciabattoni ${ }^{8}$, \\ Marina Bortul ${ }^{3}$

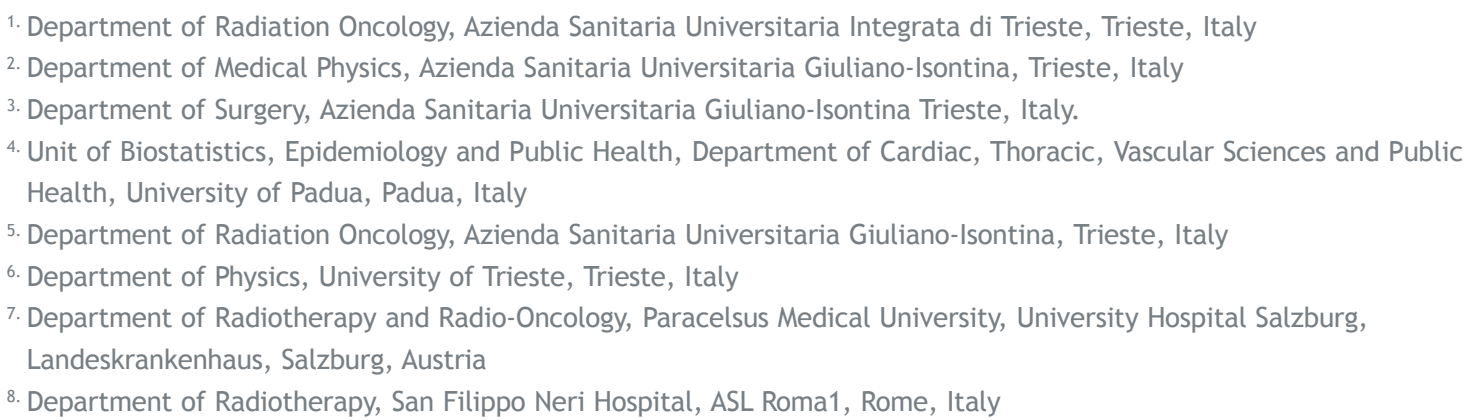

Radiol Oncol 2021; 55(3): 333-340.

Received 12 November 2020

Accepted 6 April 2021

Correspondence to: Dr. Cristiana Vidali, former Senior Assistant Department of Radiation Oncology, Azienda Sanitaria Universitaria Integrata di Trieste, Trieste, Italy. E-mail: cristiana.vidali@libero.it

Disclosure: No potential conflicts of interest were disclosed.

This is an open access article under the CC BY-NC-ND license (http://creativecommons.org/licenses/by-nc-nd/4.0/).

Background. Breast intraoperative electron radiation therapy (B-IOERT) can be used in clinical practice both as elective irradiation (partial breast irradiation - APBI) in low risk breast cancer patients, and as an anticipated boost. The procedure generally includes the use of a shielding disk between the residual breast and the pectoralis fascia for the protection of the tissues underneath the target volume. The aim of the study was to evaluate the role of intraoperative ultrasound (IOUS) in improving the quality of B-IOERT.

Patients and methods. B-IOERT was introduced in Trieste in 2012 and its technique was improved in 2014 with IOUS. Both, needle and IOUS were used to measure target thickness and the latter was used even to check the correct position of the shielding disk. The primary endpoint of the study was the evaluation of the effectiveness of IOUS in reducing the risk of a disk misalignment related to B-IOERT and the secondary endpoint was the analysis of acute and late toxicity, by comparing two groups of patients treated with IOERT as a boost, either measured with IOUS and needle (Group 1) or with needle alone (Group 2). Acute and late toxicity were evaluated by validated scoring systems. Results. From the institutional patients who were treated between June 2012 and October 2019, 109 were eligible for this study (corresponding to 110 cases, as one patients underwent bilateral conservative surgery and bilateral B-IOERT). Of these, 38 were allocated to group 1 and 72 to group 2. The target thickness measured with the IOUS probe and with the needle were similar (mean difference of $0.1 \mathrm{~mm}, \mathrm{p}=0.38$ ). The percentage of patients in which the shield was perfectly aligned after IOUS introduction increased from $23 \%$ to more than $70 \%$. Moreover, patients treated after IOUS guidance had less acute toxicity ( $36.8 \%$ vs. $48.6 \%, p=0.33$ ) from radiation therapy, which reached no statistical significance. Late toxicity turned out to be similar regardless of the use of IOUS guidance: $39.5 \%$ vs. $37.5 \%$ ( $p=0.99$ ). Conclusions. IOUS showed to be accurate in measuring the target depth and decrease the misalignment between collimator and disk. Furthermore there was an absolute decrease in acute toxicity, even though not statistically significant, in the group of women who underwent B-IOERT with IOUS guidance.

Key words: breast cancer; intraoperative electron radiation therapy; ultrasound; whole breast radiotherapy 


\section{Introduction}

Breast cancer is the most common malignant disease among women and about 53000 new cases were estimated in Italy in 2019. ${ }^{1}$ It still represents the first cause of death for cancer among women, even though there is a downward trend in the mortality in recent years $(0.8 \% /$ year $)$, due to the spread of screening programs and to therapeutic progress. $^{2}$

Adjuvant radiotherapy (RT) after breast conservative surgery is currently considered the standard treatment for early breast cancer and plays an important role to reduce local recurrences (LR) and to improve disease-free and overall survival. ${ }^{3}$

Traditionally whole breast radiation therapy (WBRT) consisted of a total dose of 50-50.4 Gy in 25-28 fractions. In recent years moderate hypofractionated WBRT has become the preferred dose-fractionation scheme, with a total dose of 40 Gy in 15 fractions or 42.50 Gy in 16 fractions, as loco-regional and survival endpoint analyses have demonstrated no inferiority compared to a conventionally fractionated schedule in several metaanalyses. ${ }^{4,5}$

The use of an additional external boost, of 10-16 Gy to the tumor bed can reduce the local failure rate from $10.2 \%$ to $6.2 \%(p<0.0001) .{ }^{6}$ This effect could be observed in all age-classes whereas the absolute gain is greatest in the group below 40 years. So far the impact of the boost has not yet been explicitly investigated in hypofractionation trials. ${ }^{4,5}$

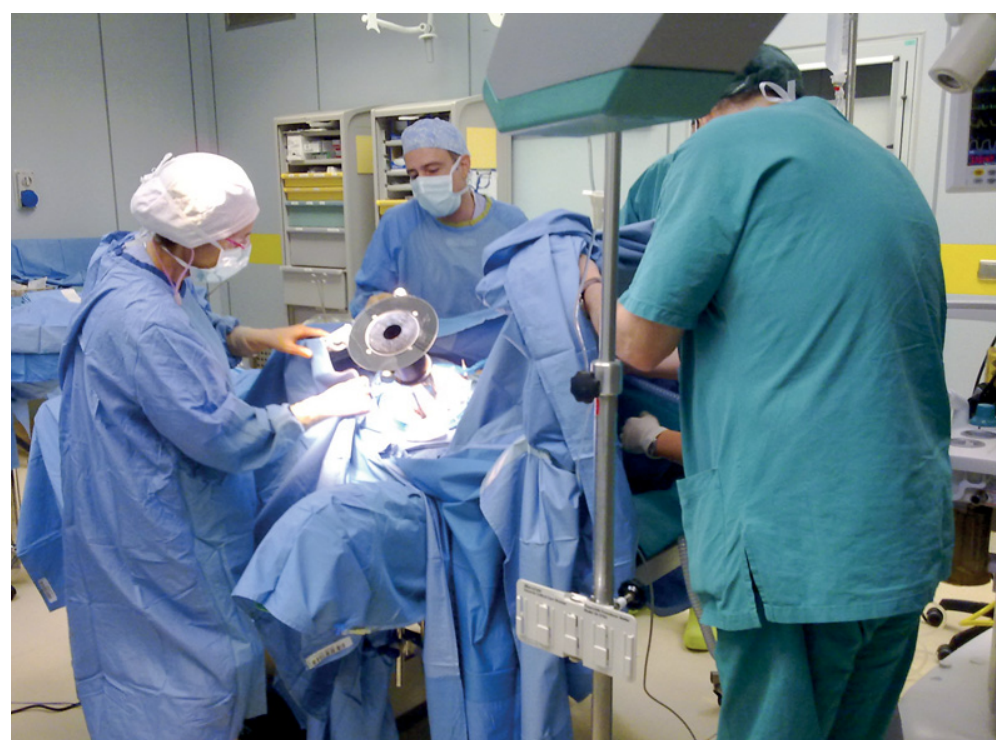

FIGURE 1. First case of breast intraoperative electron radiation therapy (B-IOERT) in Trieste using the dedicated electron beam accelerator Mobetron by IntraOp ${ }^{\circledR}$ Company.
Intraoperative electron beam radiotherapy (IOERT) in the treatment of early-stage breast cancer was introduced into the clinical practice at the end of the 1990s, when dedicated mobile linear accelerators (Linacs) became available. ${ }^{6}$ It can be used both as elective RT to the partial breast as accelerated partial breast irradiation (APBI) and as an anticipated boost. ${ }^{6-8}$ In this case, it shortens the total radiation treatment time by $1-1.5$ weeks and improves the precision of dose delivery to the tumor bed, which provides high local in- breast control rates for patients allocated to every risk group. ${ }^{8}$

Clinical data and technical details for both possible types of IOERT application, as a boost and as APBI, were summarized and discussed extensively within recently published ESTRO-guidelines. ${ }^{9}$

Beside others, in breast intraoperative electron radiation therapy (B-IOERT) the protection of the tissues underneath the target volume, as the heart, lungs, and bony structures, is obtained by positioning a shielding disk between the residual breast and the pectoralis muscle. The two main criticalities in this step of the procedure are the misalignment and the wrong orientation of the disk. ${ }^{10,11}$

B-IOERT was introduced in the "Azienda Sanitaria Universitaria Integrata" of Trieste in 2012 using the Mobetron ${ }^{\circledR}$ (IntraOp Medical, Inc. Santa Clara, CA), a "dedicated" electron beam accelerator, that produces electrons with nominal energies of 6,9 , and $12 \mathrm{MeV}$ at dose rates up to approximately $10 \mathrm{~Gy} / \mathrm{min}$ (Figure 1). Initially it was used as an anticipated boost in the treatment of early breast cancer and since March 2018 also as APBI in selected patients, according to the international recommendations. ${ }^{12}$ Its technique was improved in 2014 with IOUS. Both needle and IOUS were used to measure target thickness; the latter was used even to check the correct position of the shielding disk.

The primary endpoint of this retrospective study was the evaluation of the role of IOUS in reducing the risk of the disk misalignment related to B-IOERT and the secondary endpoint was the analysis of acute and late toxicity, by comparing two groups of patients treated with IOERT as a boost, whose target was measured either with IOUS and needle (Group 1) or with needle alone (Group 2).

\section{Patients and methods}

This is a retrospective cohort analysis of prospectively recorded data involving patients who underwent B-IOERT as an anticipated boost between 


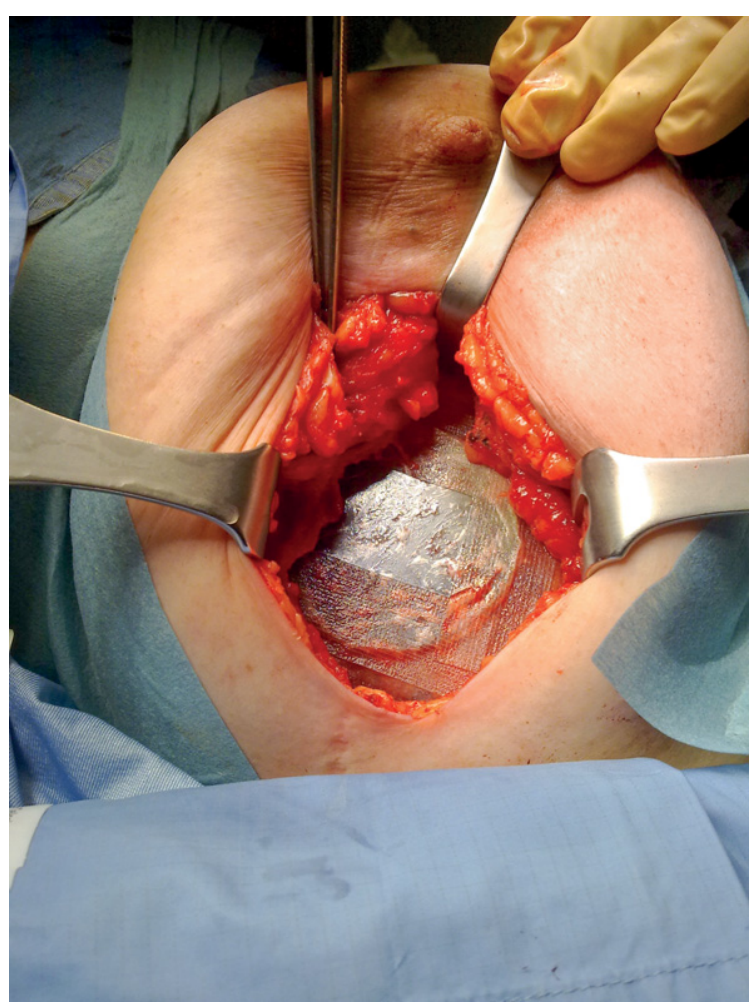

FIGURE 2A. The shielding disk positioned beneath the tumor bed during the operation.

June 2012 and November 2019 in the "Azienda Sanitaria Universitaria Integrata di Trieste".

B-IOERT boost dose prescription was $10 \mathrm{~Gy}$ specified as maximum dose; the planning target volume (PTV) should be encompassed by $90 \%$ of the prescribed dose (i.e. 9 Gy); dose inhomogeneity: $-10 \%$ within the target volume was allowed. It was followed by conventionally fractionated WBRT with a total dose of $50 \mathrm{~Gy}$ (2 Gy/25 fr.).

Since November 2014 we have taken part in the HIOB protocol (Hypofractionated WholeBreast Irradiation preceded by Intra-Operative Radiotherapy with Electrons as anticipated Boost Prospective one-armed multi-center-trial), after the approval of the Regional Ethics Committee.

The anticipated IOERT boost dose was 11,10 Gy, specified at the point of maximum dose on the central axis depth dose curve, and it was followed by hypofractionated WBRT with a total dose of 40,5 Gy in 15 fractions, 2,7 Gy/fr. ${ }^{13}$ First clinical results on treatment tolerance (toxicity and cosmetic outcome) were published in 2020. ${ }^{14}$

In the whole series before starting IOERT (either as a boost or as the unique radiation treatment), a shielding disk was positioned beneath the tumor bed on the pectoralis muscle (Figure 2A). A disk

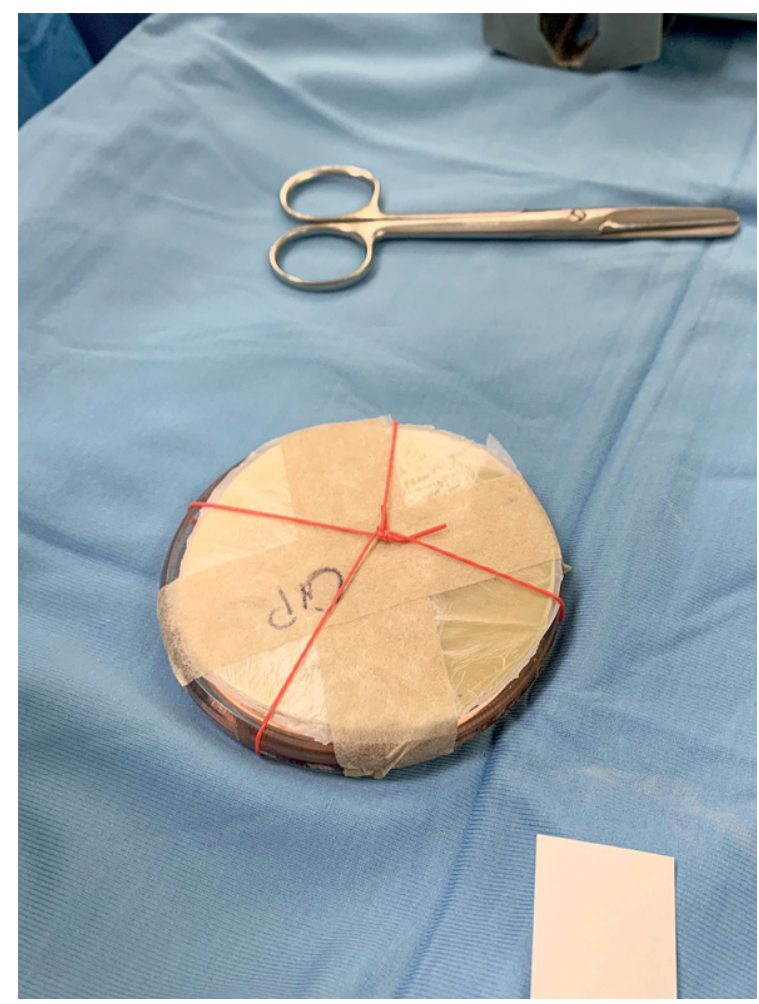

FIGURE 2B. The disk provided by IntraOp ${ }^{\circledR}$ Company, made up of three stacked layers.

provided by IntraOp ${ }^{\circledR}$ Company was employed, made by stacking a $5 \mathrm{~mm}$ polymethyl methacrylate (PMMA) layer, a $3 \mathrm{~mm}$ copper layer and $2 \mathrm{~mm}$ PMMA layer (Figure 2B). ${ }^{15}$

The optimum size of the shielding disk depends upon the electron applicator chosen for the treatment. As described in our research and in some recent studies, the set-up of normal tissue protection and applicator placement are closely correlated with two very relevant risks in IOERT treatments: misalignment and wrong orientation of the shielding disk. $11,16,17$ The low accuracy in the alignment of the disk would cause the delivery of an excess of dose to the underlying normal tissues. The surrounding healthy gland was sutured above the shielding disk.

Initially target depth was measured with a needle in 5 different points of the tumor bed and the average value was assumed in order to select the proper electron energy. Since the end of 2014, when HIOB Protocol was introduced, both needle and IOUS were used in a certain number of cases, to obtain 5 measures of the target thickness and the latter was used even to check the correct position of the shielding disk. An Esaote MyLab ${ }^{\mathrm{TM}}$ One/Touch equipment with SL 3332 linear probe was applied. 
The effectiveness of the correct localization by IOUS was verified employing "in vivo dosimetry". A properly calibrated EBT3 radiochromic film can be fixed on both faces of the disk, allowing to obtain two images that provide a detailed bidimensional dose distribution. From the first image, obtained just below the target, it is possible to measure the absolute entrance dose in that position. Moreover, the visual analysis acquired within the prepared target tissue can provide the surgeon with an effective feedback about the actual homogeneity acquired in the reconstruction of the target. From the second image positioned beyond the shielding disk, it is possible to evaluate the fraction of the dose passing through the disk and arriving at the underlying healthy tissue. ${ }^{15}$

The post-processing analysis of the dose distribution measured on the films provides a quantitative estimate of the misalignment between the collimator and the disk.

In order to investigate possible differences in the target thickness between needle and IOUS, which could alter the results, we compared preliminarily these measures in a sample of 23 patients.

Between June 2012 and October 2019 a total of 109 women (corresponding to 110 cases, as one patients underwent bilateral conservative surgery and bilateral B-IOERT for two different cancers on both breasts) underwent B-IOERT as an anticipated boost and 35 of them entered the HIOB trial. ${ }^{13,14}$ In 38 cases both needle and IOUS were used (Group 1 ) and in 72 only the needle (Group 2).

Acute toxicity was rated according to the Radiation Therapy Oncology Group (RTOG) criteria $^{18}$ and late toxicity to the LENT-SOMA score, respectively. ${ }^{19}$

This study was approved by the Institutional Review Boards of the Hospital and all patients signed preoperatively a specific informed consent.

\section{Statistical analysis}

The statistical analysis was performed by $\mathrm{R}$ software (version 3.5.0). Shapiro-Wilk test was applied to quantitative (continuous) variables to check for distribution normality. Continuous variables were reported as median with range (minimum-maximum). Qualitative (categorical) variables were reported as absolute frequencies and/or percentages. Measures of target thickness (in $\mathrm{mm}$ ) between needle and IOUS were compared by paired student's t test for every patients and overall. Moreover, Lin's concordance correlation coefficient $\left(\rho_{\mathrm{c}}\right)$ was calculated to evaluated the agreement on target thick- ness obtained by the two methods. $\rho_{\mathrm{c}}$ ranges from 0 to 1 , with perfect agreement at 1 while values near to 0 indicates no agreement. The value of ca $\rho_{\mathrm{c}}$ was interpreted according to the Mc-Bride classification (< 0.90: poor; 0.90-0.95: moderate; 0.95-0.99: substantial; > 0.99: almost perfect). Categorical variables were compared by Chi-square test or Fischer's exact test whenever appropriate. All p-values were calculated from 2-sided tests using 0.05 as the significance level.

\section{Results}

\section{Description of the population}

Information on patient characteristics and adjuvant systemic treatments of the whole population and of the two groups are summarized in Table 1. Baseline characteristics are similar between treatment groups. The median age of the entire cohort of patients was 67 years (range: 43-85).

Two patients (one belonged to Group 1 and the other to Group 2) refused adjuvant radiotherapy and one (Group 2) performed it elsewhere.

Of note, two patients did not receive the allocated systemic therapy: one was discouraged for endocrine therapy due to severe osteoporosis and the second refused chemotherapy without compensating it by another oncological relevant medication.

With a median follow-up of 52 months (range: 5-87), 3 patients died of breast cancer and 4 of other causes; one patient is alive with progressive disease and another one was treated for a contralateral tumor and is disease-free at the last follow-up.

\section{Effect of IOUS}

The target thickness detected preliminarily with IOUS in a sample of 23 patients turned out to be completely in agreement with the measures obtained with the needle, with Lin's concordance coefficient $\rho_{\mathrm{c}}=0.98,95 \% \mathrm{CI}$ : 0,96:0.99 and with a negligible average difference of $0.1 \mathrm{~mm}$ (range $0.1-1.2$ $\mathrm{mm})(\mathrm{p}=0.38)$ as described in Supplementary Table 1.

The EBT3 "in vivo dosimetry" showed that IOUS guidance achieved a reduction in the misalignment with an electron field area outside the shielding disk reduction from $5.6 \mathrm{~cm}^{2}$ to $2.6 \mathrm{~cm}^{2}$.

After IOUS introduction, the percentage of patients in whom the shield was perfectly aligned, defined as a field totally inside the shield, increased from $23 \%$ to more than $70 \%$. The proper 
TABLE 1. Description of the population

\begin{tabular}{|c|c|c|c|c|}
\hline Variables & All cohort $(n=110)$ & Group $1(n=38)$ & Group $2(n=72)$ & $p$-value \\
\hline Age (years) Median (range) & $67(43-85)$ & $67(48-80)$ & $67(43-85)$ & $p=0.51$ \\
\hline $\begin{array}{l}\text { Clinical stage } \\
\text { cTla NO } \\
\text { cTlb NO } \\
\text { cTlc NO } \\
\text { cTlb N1 } \\
\text { cT2 NO }\end{array}$ & $\begin{aligned} 2 & (1.8 \%) \\
47 & (42.8 \%) \\
59 & (53.6 \%) \\
1 & (0.9 \%) \\
1 & (0.9 \%)\end{aligned}$ & $\begin{array}{c}1(2.6 \%) \\
20(52.6 \%) \\
17(44.8 \%) \\
0(0.0 \%) \\
0(0.0 \%)\end{array}$ & $\begin{aligned} 1 & (1.4 \%) \\
27 & (37.5 \%) \\
42 & (58.3 \%) \\
1 & (1.4 \%) \\
1 & (1.4 \%)\end{aligned}$ & $p=0.42$ \\
\hline $\begin{array}{l}\text { Histological type } \\
\text { Ductal carcinoma in situ (DCIS) } \\
\text { Invasive ductal carcinoma } \\
\text { Invasive lobular carcinoma } \\
\text { Others }\end{array}$ & $\begin{array}{c}1(0.9 \%) \\
70(63.6 \%) \\
19(17.3 \%) \\
20(18.2 \%)\end{array}$ & $\begin{array}{r}1(2.6 \%) \\
26(68.4 \%) \\
5(13.2 \%) \\
6(15.8 \%)\end{array}$ & $\begin{array}{c}0(0.0 \%) \\
44(61.1 \%) \\
14(19.45 \%) \\
14(19.45 \%)\end{array}$ & $p=0.46$ \\
\hline Multifocal disease & $6(5.4 \%)$ & $4(10.5 \%)$ & $2(2.8 \%)$ & $p=0.21$ \\
\hline $\begin{array}{l}\text { Pathological Stage pT } \\
\text { pTis } \\
\text { PT1a } \\
\text { pT1b } \\
\text { PT1c } \\
\text { PT2 }\end{array}$ & $\begin{aligned} 1 & (0.9 \%) \\
3 & (2.7 \%) \\
41 & (37.3 \%) \\
59 & (53.6 \%) \\
6 & (5.5 \%)\end{aligned}$ & $\begin{aligned} 1 & (2.6 \%) \\
0 & (0.0 \%) \\
16 & (42.1 \%) \\
20 & (52.6 \%) \\
1 & (2.6 \%)\end{aligned}$ & $\begin{array}{c}0(0.0 \%) \\
3(4.2 \%) \\
25(34.7 \%) \\
39(54.2 \%) \\
5(6.9 \%)\end{array}$ & $p=0.40$ \\
\hline $\begin{array}{l}\text { Pathological Stage pN } \\
\text { pNO } \\
\text { pN1mi } \\
\text { pNla } \\
\text { pN3 }\end{array}$ & $\begin{array}{c}88(80.0 \%) \\
7 \text { (6.4\%) } \\
14(12.7 \%) \\
1(0.9 \%)\end{array}$ & $\begin{array}{c}32(84.2 \%) \\
3(7.9 \%) \\
3(7.9 \%) \\
0(0.0 \%)\end{array}$ & $\begin{array}{c}56 \text { (77.8\%) } \\
4(5.6 \%) \\
11(15.3 \%) \\
1(1.4 \%)\end{array}$ & $p=0.71$ \\
\hline $\begin{array}{l}\text { Grading } \\
\text { G1 } \\
\text { G2 } \\
\text { G3 } \\
\text { Gx }\end{array}$ & $\begin{array}{r}15(13.7 \%) \\
68(61.8 \%) \\
21(19.1 \%) \\
6(5.4 \%)\end{array}$ & $\begin{array}{r}7(18.4 \%) \\
24(63.2 \%) \\
6(15.8 \%) \\
1 \quad(2.6 \%)\end{array}$ & $\begin{array}{c}8 \text { (11.1\%) } \\
44(61.1 \%) \\
15(20.8 \%) \\
5(7 \%)\end{array}$ & $p=0.67$ \\
\hline $\begin{array}{l}\text { Adjuvant therapy* } \\
\text { None } \\
\text { Chemotherapy } \\
\text { Endocrine therapy } \\
\text { Chemotherapy + Endocrine therapy } \\
\text { Chemotherapy + Trastuzumab }\end{array}$ & $\begin{array}{l}2(1.8 \%) \\
6(5.5 \%) \\
85(78 \%) \\
12(11 \%) \\
4(3.7 \%)\end{array}$ & $\begin{array}{c}0(0.0 \%) \\
2(5.4 \%) \\
29(78.4 \%) \\
4(10.8 \%) \\
2(5.4 \%)\end{array}$ & $\begin{array}{c}2(2.8 \%) \\
4(5.6 \%) \\
56(77.7 \%) \\
8(11.1 \%) \\
2(2.8 \%)\end{array}$ & $p=0.92$ \\
\hline
\end{tabular}

* Calculated on 109 patients

alignment with IOUS improved the absolute dose accuracy and tripled the number of patients without disk misalignment. The results of post processing analysis of the dose distribution measured on film, the collimator used, the estimated dose, the area outside the shielding disk and the dose map distribution are presented in Figure 3.

In the whole series acute toxicity, detected 4 weeks after WBRT, occurred in 49 patients ( $44.5 \%)$ : G1 in $36(32.7 \%)$ and G2 in $13(11.8 \%)$ of them. The most common acute reaction was erythema due to postoperative WBRT. As perioperative complications the occurrence of seroma (one case), hematoma ( 2 cases) and lymphocele ( 2 cases) were identified. With regard to clinical collateral effects, patients allocated to group 1 developed less radiation induced acute toxicity compared to group $2(36.8 \%$

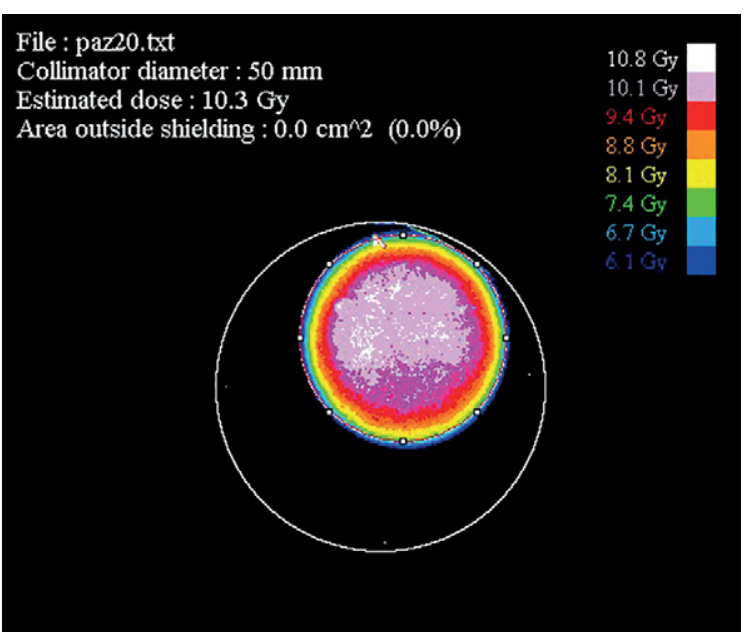

FIGURE 3. Post processing analysis of the dose distribution measured on film. 
TABLE 2. Acute toxicity of radiotherapy in the two groups

\begin{tabular}{lccc}
\hline & Group 1 $(\mathrm{n}=\mathbf{3 8})$ & Group 2 $(\mathrm{n}=\mathbf{7 2})$ & Total $(\mathrm{n}=110)$ \\
\hline Grade 0 & $23(60.5 \%)$ & $36(50 \%)$ & $59(53.6 \%)$ \\
Grade 1 & $8(21.1 \%)$ & $28(38.9 \%)$ & $36(32.7 \%)$ \\
Grade 2 & $6(15.8 \%)$ & $7(9.7 \%)$ & $13(11.8 \%)$ \\
Grade X & $1(2.6 \%)$ & $1(1.4 \%)$ & $2(1.9 \%)$ \\
Grade 1-2 & $14(36.8 \%)$ & $35(48.6 \%)$ & $49(44.5 \%)$
\end{tabular}

Group 1 = cases treated with breast intraoperative electron radiation therapy (B-IOERT) as a boos in which both needle and intraoperative ultrasound (IOUS) were used; Group 2 = cases treated with B-IOERT as a boost in which only the needle was used

TABLE 3. Late toxicity of radiotherapy in the two groups

\begin{tabular}{lccc}
\hline & Group 1 $(\mathbf{n = 3 8 )}$ & Group 2 $(\mathbf{n}=\mathbf{7 2})$ & Total $(\mathbf{n = 1 1 0 )}$ \\
\hline Grade 0 & $22(57.9 \%)$ & $43(59.7 \%)$ & $65(59.1 \%)$ \\
Grade 1 & $12(31.6 \%)$ & $24(33.3 \%)$ & $36(32.7 \%)$ \\
Grade 2 & $3(7.9 \%)$ & $3(4.2 \%)$ & $6(5.5 \%)$ \\
Grade X & $1(2.6 \%)$ & $2(2.8 \%)$ & $3(2.7 \%)$ \\
Grade 1-2 & $15(39.5 \%)$ & $27(37.5 \%)$ & $42(38.2 \%)$
\end{tabular}

Group 1 = cases treated with breast intraoperative electron radiation therapy (B-IOERT) as a boost in which both needle and intraoperative ultrasound (IOUS) were used; Group 2 = cases treated with B-IOERT as a boost in which only the needle was used

vs $48.6 \%)$, which turned out as not statistically significant $(\mathrm{p}=0.33)$ (Table 2).

Late toxicity, evaluated at the last follow-up, was observed in 42 patients (Grade 1: 36 cases; Grade 2: 6 cases). There was a negligible difference in the two groups of patients: $39.5 \%$ (Group 1) vs. $37.5 \%$ (Group 2) $(\mathrm{p}=0.99)$. (Table 3). The most common type of sequelae were: fibrosis/fat necrosis (G1/2: $21.8 \%$ ) and breast edema (G1/2: $6.4 \%)$. In the whole population no Grade 3 acute and late toxicity occurred.

Detailed results for late toxicity according to LENT-SOMA score are summarized in Supplementary Table 2. As regards the different WBRT schedules, no significant differences were detected for acute and late toxicity (Supplementary Table 3A, 3B).

\section{Discussion}

The primary objective of this analysis was to evaluate the role of IOUS in improving the quality and in reducing the risks related to B-IOERT.

We could demonstrate that IOERT is a safe treatment modality; the target thickness measures performed with the needle were comparable with those obtained with IOUS. Moreover IOUS contributed considerably to optimize the alignment of the shielding disk.

At present, a commercially available treatment planning system (TPS) for IOERT, which allows to accurately illustrate a dose distribution within the target as well as the surrounding tissues, is used only by a small number of Centers. ${ }^{20}$ The process leading to intraoperative radiation treatment, without TPS, is mostly the result of a sequence of manually handled actions involving the Surgeon, the Radiation Oncologist, the Medical Physicist, the Technicians and the Nurses of the operating room..$^{9,11}$

Some papers examined the importance of risk assessment analysis applied to IOERT procedure: in the Italian publications a dedicated mobile Linac was used, while in the Spanish analysis the irradiation was performed with a conventional Linac. ${ }^{11,16,17}$ In all of the studies the highest risk was associated with the alignment of the shielding disk, as it was observed in our experience.

The low accuracy in the alignment of the disk would cause the delivery of an excess of dose to the underlying normal tissues and is mainly related to the lack of direct visual disk control. ${ }^{11}$ Selecting a plate much larger than the applicator size and fixing it to the pectoralis fascia is some effective corrective actions. The introduction of intraoperative ultrasound allows to check the position of the shielding disk as well as to better define the target thickness and is an important strategy for reducing the risk of shield misalignment and incorrect thickness measures.

The secondary endpoint of the study was the evaluation of early and late toxicity in the whole series with the comparison between the group of patients who underwent IOUS examination vs. the group who underwent only needle measures.

The target volume with IOERT boost is smaller than the volume with conventional EBRT boost. This leads to a reduction in the radiation exposure of the subcutaneous tissues and intra-thoracic organs (i.e. lungs and heart). Besides it avoids the irradiation of the skin and of the contralateral mammary gland, reducing both the short-term and long-term sequelae correlated with RT and resulting in good cosmesis. ${ }^{9}$

To date various papers have been published on IOERT boost in the treatment of early-stage breast cancer, with the largest deriving from a pooled analysis of the International Society of Intraoperative Radiation Therapy (ISIORT) 
Europe, which was updated for the Salzburg cohort with a 10 year follow-up in 2018..$^{21,22}$ Excellent local control was reported in all the studies and side effects were generally low and acceptable. Merrick et al. reported $11 \%$ delayed fibrosis around the tumor bed and late breast or arm pain in $8 \%$ of patients; Lemanski et al. Grade 2 subcutaneous fibrosis in $14 \%$ and late breast or arm pain in $8 \%$ of patients. ${ }^{23,24}$ In the Salzburg experience less than $2 \%$ of patients developed G3 late toxicity. ${ }^{8}$ In the prospective study by Wong et al., there was no G3 acute toxicity; two patient developed G3 late toxicity $(3.8 \%)$ and 5 patients $(9.6 \%)$ fat necrosis. ${ }^{25} \mathrm{In}$ these studies conventionally fractionated WBRT (total dose: 50-56 Gy/25-28 fr.) was delivered. In a retrospective German study 14 of 157 patients $(8.9 \%)$ were treated with hypofractionated WBRT; the whole acute skin toxicity was evaluable in 153 patients: G1 in $75.2 \%$; G2 in $15.7 \%$ and G3 in 4.6\%, 6-8 weeks after WBRT. ${ }^{26}$

In three prospective investigations, IOERT boost was combined with hypofractionated WBRT. In the report by Ivaldi et al. G1 and G2 acute toxicity were found in 79/132 (59.8\%) and in 22/132 (16.7\%) respectively, and G3 in 3/132 (2.3\%) patients one month after the end of WBRT. ${ }^{27}$ The recorded late skin toxicity was Grade 4 in one patient, Grade 3 in another one and Grade 2 or less in 106/108 cases $(98.2 \%)$ with a median follow-up of 11 months. In the study by Bhandari et al. only 24 patients underwent IOERT as a boost; after a short follow-up (12 months), G1 and G2 acute toxicity was present in $47.8 \%$ and $4.4 \%$ of the cases, respectively; late toxicity in $40 \%$ (G1) and in $10 \%$ (G2) of the cases. ${ }^{28}$ In the recent paper from $\mathrm{HIOB}$ prospective multicenter trial, G0-1 acute effects were noted in $92 \%$ of patients and G3 in one patient, 4 weeks after the end of WBRT; G0-1 late toxicity in 93\%, G2 in 4.3\% and G3-4 in $2.7 \%$ of cases at 4 years. ${ }^{14}$

Our results are similar to the data reported in the other series and even better than the results of the early published reports. Additionally the effectiveness of IOUS application during IOERT procedure was analyzed and less acute toxicity, even if not statistically significant, was observed in the group of women who underwent B-IOERT with IOUS guidance. As regards G1-G2 late toxicity, no differences in the two groups of patients were detected. No grade 3 and 4 acute and late toxicity were present in both treatment groups.

To our knowledge no other studies evaluated shielding disk misalignment and possible correlations with acute or late toxicity in B-IOERT procedure. However, these results should be taken with caution. First of all this is a retrospective study; besides the analysis was carried out on a small series of patients and median follow-up is rather short. A longer follow-up is advisable, to monitor the incidence of late toxicity.

\section{Conclusions}

In this study, IOUS turned out to be an excellent intraoperative imaging modality that allows to measure the thickness of the tumor bed, to optimize the position of the shielding disk and it possibly contributes to decrease adverse effects in term of acute toxicity. Its application is advisable also in other areas of intraoperative radiation treatments.

\section{References}

1. AIOM-AIRTum. [The number of cancer in Italy 2019]. [Italian]. Brescia: Intermedia Editore; 2019. Available at: www.medinews.it

2. AIOM Linee guida. [Breast neoplasms, 2019]. [Italian]. [internet]. 2019. [cited 2020 Oct 14]. Available at: https://www.aiom.it/linee-guida-aiomneoplasie-della-mammella-2019

3. Early Breast Cancer Trialists' Collaborative Group (EBCTCG). Effect of radiotherapy after breast-conserving surgery on 10-year recurrence and 15-year breast cancer death: meta-analysis of individual patient data for 10,801 women in 17 randomized trials. Lancet 2011; 378: 1707-16. doi: 10.1016/ S0140-6736(11)61629-2

4. Hickey BE, James ML, Lehman M, Hider PN, Jeffery M, Francis DP, et al. Fraction size in radiation therapy for breast conservation in early breast cancer. Cochrane Database Syst Rev, 2016; 7: CD003860. doi: 10.1002/14651858.CD003860.pub4.

5. Valle LF, Agarwal S, Bickel KE, Herchek HA, Nalepinski DC, Kapadia NS. Hypofractionated whole breast radiotherapy in breast conservation for early-stage breast cancer: a systematic review and meta-analysis of randomized trials. Breast Cancer Res Treat 2017; 162: 409-17. doi: 10.1007/ s10549-017-4118-7

6. Veronesi U, Orecchia R, Maisonneuve P, Viale G, Rotmensz N, Sangalli C, et al. Intraoperative radiotherapy versus external radiotherapy for early breast cancer (ELIOT): a randomised controlled equivalence trial. Lancet Oncol 2013; 14: 1269-77. doi: 10.1016/S1470-2045(13)70497-2

7. Maluta S, Dall'Oglio S, Goer DA, Marciai N. Intraoperative electron radiotherapy (IOERT) as an alternative to standard whole breast irradiation: only for low-risk subgroups? Breast Care 2014; 9: 102-6. doi: 10.1159/000362392

8. Sedlmayer F, Reitsamer R, Wenz F, Sperk E, Fussl C, Kaiser J, et al. Intraoperative radiotherapy (IORT) as boost in breast cancer. Radiat Oncol 2017; 12: 23. doi: 10.1186/s13014-016-0749-9

9. Fastner G, Gaisberger C, Kaiser J, Scherer P, Ciabattoni A, Petoukhova A, et al. ESTRO IORT Task Force/ACROP recommendations for intraoperative radiation therapy with electrons (IOERT) in breast cancer. Radiother Oncol 2020; 149: 150-15. doi: 10.1016/j.radonc.2020.04.059

10. Russo G, Casarino C, Arnetta G, Candiano G, Stefano A, Alongi F, et al. Dose distribution changes with shielding disc misalignments and wrong orientations in breast IOERT: a Monte Carlo-GEANT ${ }_{4}$ and experimental study. J Appl Clin Med Phys 2012; 13: 74-92. doi: 10.1120/jacmp.v13i5.3817

11. Vidali C, Severgnini M, Urbani M, Toscano L, Perulli A, Bortul M. FMECA application to intraoperative electron beam radiotherapy procedure as a quality method to prevent and reduce patient's risk in conservative surgery for breast cancer. Front Med 2017; 4: 138. doi: 10.3389/fmed.2017.00138 
12. Correa C, Harris EE, Leonardi MC, Smith BD, Taghian AG, Thompson AM, et al. Accelerated partial breast irradiation: executive summary for the update of an ASTRO evidence-based consensus statement. Pract Radiat Oncol 2017; 7: 73-9. doi: 10.1016/j.prro.2016.09.007

13. Sedlmayer F, Fastner G, Sedlmayer F. Intra-operative electron boost and hypofractionated whole-breast irradiation during breast-conserving treatment (BCT) (HIOB). ClinicalTrials.gov Identifier: NCT01343459. [internet]. [cited 2020 Oct 13]. Available at: https://clinicaltrials.gov/ct2/show/NCT01343459

14. Fastner G, Reitsamer R, Urbanski B, Kopp P, Murawa D, Adamczyk B, et al. Toxicity and cosmetic outcome after hypofractionated whole breast irradiation and boost-IOERT in early stage breast cancer (HIOB): first results of a prospective multicenter trial (NCT01343459). Radiother Oncol 2020; 146: 136-42. doi: 10.1016/j.radonc.2020.02.001

15. Severgnini M, de Denaro M, Bortul M, Vidali C, Beorchia A. In vivo dosimetry and shielding disk alignment verification by EBT3 GAFCHROMIC film in breast IOERT treatment. J Appl Clin Med Phys 2015; 16: 112-20. doi: 10.1120/jacmp.v16i1.5065

16. Ciocca, M, Cantone MC, Veronese I, Cattani F, Pedroli G, Molinelli S, et al. Application of failure mode and effects analysis to intraoperative radiation therapy using mobile electron linear accelerators. Int J Radiat Oncol Biol Phys 2012; 82: e305-11. doi: 10.1016/j.jijobp.2011.05.010

17. López-Tarjuelo J, Bouché-Babiloni A, Santos-Serra A, Morillo-Macías V, Calvo FA, Kubyshin Y, et al. Failure mode and effect analysis oriented to risk-reduction interventions in intraoperative electron radiation therapy: the specific impact of patient transportation, automation, and treatment planning availability. Radiother Oncol 2014; 113: 283-9. doi: 10.1016/j. radonc.2014.11.012

18. Cox JD, Stetz J, Pajak TF. Toxicity criteria of the Radiation Therapy Oncology Group (RTOG) and the European Organization for Research and Treatment of Cancer (EORTC). Int J Radiat Oncol Biol Phys 1995; 31: 1341-6. doi: 10.1016/0360-3016(95)00060-C

19. Rubin P, Constine LS, Fajardo LF, Phillips TF, Wasserman TH. RTOG Late Effects Working Group. Overview of late effects of normal tissues (LENT) scoring system. Int J Radiat Oncol Biol Phys 1995; 31: 1041-2. doi: 10.1016/0360-3016(95)00057-6

20. Valdivieso-Casique MF, Rodríguez R, Rodríguez-Bescós $S$, Lardíes D, Guerra $\mathrm{P}$, Ledesma MJ, et al. RADIANCE - a planning software for intra-operative radiation therapy. Transl Cancer Res 2015; 4: 196-209. doi: 10.3978/j. issn.2218-676X.2015.04.05

21. Fastner G, Sedlmayer F, Merz F, Deutschmann H, Reitsamer R, Menzel C, et al. IORT with electrons as boost strategy during breast conserving therapy in limited stage breast cancer: long term results of an ISIORT pooled analysis. Radiother Oncol 2013; 108: 279-86. doi: 10.1016/j.radonc.2013.05.031

22. Kaiser J, Reitsamer R, Kopp P, Gaisberger C, Kopp M, Fischer T, et al Intraoperative electron radiotherapy (IOERT) in the treatment of primary breast cancer. Breast Care 2018; 13: 162-7. doi: 10.1159/000489637

23. Merrick HW 3rd, Hager E, Dobelbower RR Jr. Intraoperative radiation therapy for breast cancer. Surg Oncol Clin N Am 2003; 12: 1065-78. doi: 10.1016/s1055-3207(03)00098-x

24. Lemanski C, Azria D, Thezenas S, Gutowski M, Saint-Aubert B, Rouanet $P$, et al. Intraoperative radiotherapy given as a boost for early breast cancer: long-term clinical and cosmetic results. Int J Radiat Oncol Biol Phys 2006; 64: 1410-5. doi: 10.1016/j.jjrobp.2005.10.025

25. Wong WW, Pockaj BA, Vora SA, Halyard MY, Gray RJ, Schild SE Six-year outcome of a prospective study evaluating tumor bed boost with intraoperative electron irradiation followed by whole-breast irradiation for earlystage breast cancer. Breast J 2014; 20: 125-30. doi:10.1111/tbj.12235

26. Konig L, Lang K, Heil J, Golatta M, Major G, Krug D, et al. Acute toxicity and early oncological outcomes after intraoperative electron radiotherapy (IOERT) as boost followed by whole breast cancer patients - first clinical results from a single center. Front Oncol 2019; 9: 384. doi: 10.3389/ fonc. 2019.00384

27. Ivaldi GB, Leonardi MC, Orecchia R, Zerini D, Morra A, Galimberti V, et al. Preliminary results of electron intraoperative therapy boost and hypofractionated external beam radiotherapy after breast-conserving surgery in premenopausal women. Int J Radiat Oncol Biol Phys 2008; 72: 485-93. doi: 10.1016/j.jijrobp.2007.12.038
28. Bhandari T, Babaran W, Forouzannia A, Williams V, Harness J, Carpenter M, et al. A prospective phase I comparison of toxicity and cosmesis outcomes of single-fraction IORT and hypofractionated radiotherapy with IORT boost in early-stage breast cancer. Brachytherapy 2017; 16: 1232-1238.e2. doi: 10.1016/j.brachy.2017.09.002 